\title{
Study on Map Reading Skill of High School Students in Chuxiong City Jiaofeng Chen ${ }^{1, a}$ and Wujun $\mathrm{Xi}^{1, \mathrm{~b}}$
}

\author{
${ }^{1}$ School of Geography and Tourism Management, Chuxiong Normal University, Chuxiong, China \\ a1920548590@qq.com, babsxwj@163.com
}

Keywords: High school students; Map reading skill; Chuxiong city; Teaching strategies

\begin{abstract}
To understand the status of Map Reading Skill of high school students in Chuxiong city, reduce blindness of geography teaching improve teaching quality and efficiency, a map reading skill questionnaire survey of four high schools in Chuxiong city was made. Some problems were found as follows: the basic of geography knowledge of high school students was weak, many students lacked of interest in learning geography some students were weary of geography studying. Their geography learning abilities were weak, especially map reading skill. So, the following countermeasures and suggestions were put forward, the teachers and students should cultivate the interest in map, consolidate the basic of map knowledge of high school students, form a good habit of using map, improve the ability of using map, and create a good map learning environment.
\end{abstract}

\section{楚雄市城区高中生地理读图能力分析}

\author{
陈娇芬 $^{1, a}$, 席武俊 ${ }^{1, b}$ \\ 1. 楚雄师范学院地理科学与旅游管理学院, 中国云南楚雄市 675000 \\ a1920548590@qq.com, babsxwj@163.com
}

摘要: 为了解楚雄市城区高中生地理读图能力现状, 提高对楚雄市城区高中学生地理读图能 力, 减少地理教学的盲目性, 就楚雄市城区高中生“地理学习动机”、“地理学习兴趣”、

“地理读图现状” 等方面开展调查分析, 得出以下结论：楚雄市城区高中生地理基础知识较 弱, 对地理学习的兴趣薄弱, 甚至有厌学的的情绪; 楚雄市城区高中生地理学习能力较弱, 且以地理的读图能力薄弱最为突出。针对存在的问题, 提出以下建议: 激发学习动机, 培养 学生对地图的兴趣; 对学生的地图基础知识加以巩固; 培养学生良好的用图习惯; 培养学生 地图的应用能力; 给学生创建良好的地理地图学习环境。

关键词: 楚雄市; 高中生; 地理读图能力; 教学对策

\section{1. 引言}

地图是以注记、符号等多种形式来表达丰富的地理信息, 是地理知识的一种直观的表达, 使 学者很容易从地图中找到地理知识的规律性。学会读图是学习地理的一大特点, 它能培养学 生地理学习的兴趣, 地图与地理教学二者不可缺一, 这也是地理教学的一大特色。地理读图 能力是指从地理图像中掌握信息的能力。地图构成包括图形、数学、辅助、补充说明等几个 方面的要素。地图包含着丰富的知识点, 地理特点、规律需要通过观察、分析地图得出。养 成良好的读图习惯和较高的读图能力有助于培养学生的空间概念和空间想象力, 是学好地理 的关键。

世界各国非常重视地理读图教学, 都制定本国国家地理课程标准。例如美国和英国的国家地 理课程标准中对涉及地理读图教学的要求 ${ }^{[1]}$ 。美国威特金 ${ }^{[2]}$ 、美国著名发展心理学家加德纳 ${ }^{[3]}$ 、 美国学者丹尼斯 - 伍德 ${ }^{[4]}$ 分别从不同角度分析了空间思维、空间智力、地图的力量等内容。 我国中学地理教学大纲提出: “教师要有计划指导学生阅读地图, 掌握运用地图解释地理问 题的一些方法, 使学生形成空间观念 ${ }^{[5]}$ 。” 国内学者也从不同角度对中学地理读图能力进行 
了研究, 例如培养形象思维, 进行创新性想象 ${ }^{[6]}$; 重视地理课本插图 ${ }^{[7]}$; 强调地理学习观察法 ${ }^{[8]}$; 掌握地图的基本特征 ${ }^{[9]}$; 将教材插图和地图有机结合起来、开展填图训练提高读图能力 ${ }^{[10]}$ 等。

现代社会非常需要要求学校在教育中把促进学生的学习发展放在重要地位, 高中课程改革正 蓬勃开展, 转变学习方式是地理课程改革的显著特征。但楚雄市高中地理教学及学生读图能 力依然存在一些问题, 基于此, 对楚雄市城区高中生的地理读图能力现状（主要包括地理图 形观察能力、地理地图获取知识能力、运用地图分析问题、解决问题等方面）进行调查，同 时对调查结果进行分析, 在分析的基础上提出建议和对策以解决高中生地理读图能力出现的 问题。

\section{2. 调查对象与方法}

2.1. 调查对象

2014 年 10 月 13 日至 2014 年 12 月 20 日间对楚雄师范学院附中、龙江中学和楚雄州民族中 学进行高中生地理读图能力现状进行抽样调查。调查的楚雄师院附中是一所州属完全中学, 高中部各年级有八个班高中年级学生有 1156 人。龙江中学, 原名楚雄四中, 现高中各年级有 八个班级, 学生有 1138 人。楚雄州民族中学高中各年级有十个班级, 有 1528 人。

2. 2 . 问卷制定

问卷的制定是结合了相关学者的问卷调查内容及想要了解学生对地理读图能力某些方面的信 息而制定。本文调查研究的问卷调查共 27 道题目, 包括选择、简答两种题型。

2.3. 调查过程

问卷调查时间为 2014 年 12 月。采用匿名方式。此次调查向楚雄师范学院附中发出问卷 200 份，回收问卷 200 份，有效问卷 195 份，问卷回收率 $100 \%$ 。龙江中学发出问卷 50 份，回收 问卷 50 份, 有效问卷 50 份, 问卷回收率 $100 \%$ 。楚雄州民族中学发出问卷 50 份, 回收问卷 50 份, 有效问卷 50 份, 回收率 $100 \%$ 。在问卷调查期间, 对学生、教师进行访谈, 访谈内容 基于问卷内容进行的，有利于高中生地理读图能力现状调查分析的完整性。

\section{3. 楚雄市城区高中生地理读图能力现状调查结果与分析}

3.1. 对地理课的兴趣

从图 1 (a) 中可以看出, 三所中学的高中生对地理课兴趣一般的占多数, 其次是很感兴趣的 占少部分, 接着仍然不感兴趣的学生所占比例最少。

3.2. 是否喜欢读图题

从图 1 (b) 中可以看出, 绝大部分的学生不太喜欢读图题, 其次有一部分学生喜欢读图题, 有少部分学生很喜欢读图题, 极少部分学生从来不做读图题的。

3. 3. 学习地理是否有 “左图右书” 的习惯

学生在学习地理时是否有 “左图右书” 的习惯影响着学生的地理读图能力。从图 1(c) 中可 以看出, 楚雄师院附中、龙江中学和楚雄州民族中学高中生在学习地理时有时会用 “左图右 书” 的学生占绝大部分, 楚雄师院附中的高中生占 $19.5 \%$, 龙江中学高中生占 $18 \%$, 楚雄州民 族中学占 $8 \%$, 。没有 “左图右书” 习惯学生楚雄师院附中占 12.3 , 龙江中学学生占的很少, 有 $4 \%$ 的高中生没有 “左图右书” 的习惯，楚雄州民族中学占 $34 \%$ 。 


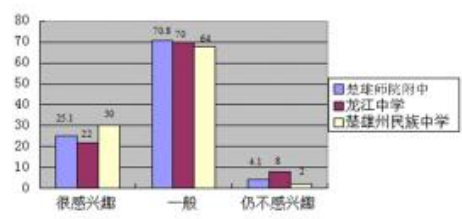

（a）高中生对地理课的兴趣

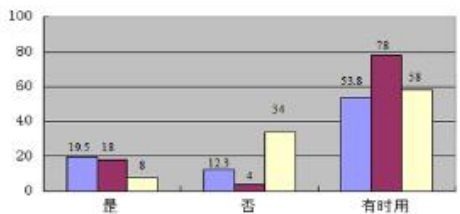

（c）学习地理是否有 “左图右书” 的习惯

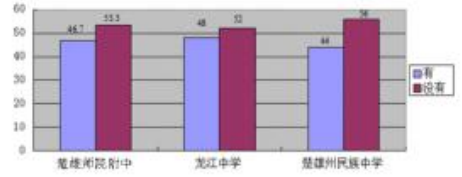

（e）日常生活中是否有用图的习惯

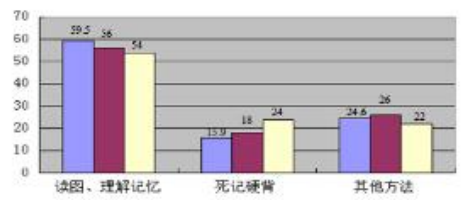

（g）记忆地理知识的方法

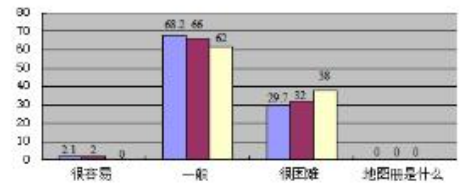

（i）是否可以不借助教材在地图册上完成填图

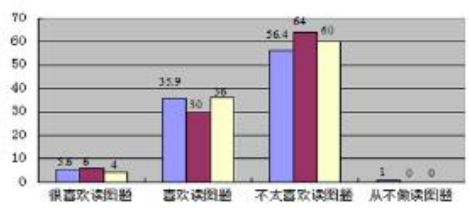

(b) 是否喜欢读图题

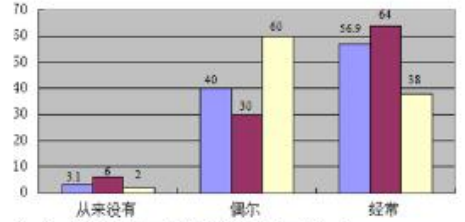

（d）老师是否经常教读图方法

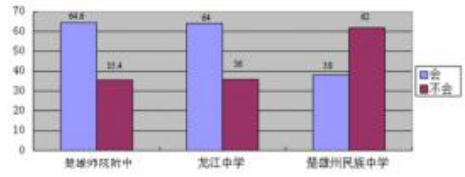

（f）是否会绘制简单的地图

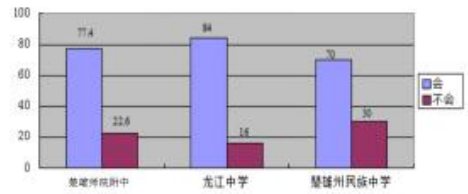

(h) 做读图填空练习时是否会选择合适的地图查阅

图 1 问卷调查结果统计图

\section{4. 老师是否经常教读图方法}

从图 1（d）中可以看出, 楚雄师院附中、龙江中学和楚雄州民族中学有绝大部分学生回答老 师经常教读图方法, 其次是老师偶尔教高中生读图方法, 极少部分高学生认为老师从来没有 教读图方法。

3.5. 日常生活中是否有用图的习惯

从图 1 (e) 中可以看出, 楚雄师院附中、龙江中学和楚雄州民族中学三所学校的高中生在日 常生活中有用图习惯所占比例都小于没有用图习惯, 这说明还有一部分学生在日常生活中还 缺乏用图的习惯。

3.6. 是否会绘制简单的地图

从图 1（f）中可以看出, 楚雄师院附中和龙江中学所调查的高中生会简单绘制地图的人数所 占比例大于不会绘制简单地图人数所占比例, 而楚雄州民族中学反之, 会绘制简单地图人数 比例小于不会绘制简单地图人数比例。

3.7. 记忆地理知识的方法

无论学习什么课程都需要靠好的学习方法来记忆课程知识, 同样地理课程知识的记忆也需要 好的、适合学生自己的学习方法来加强地理知识的学习。从图 1（g) 中可以看出, 三所学校 高中生中超过 50\%的学生都习惯用读图、理解记忆的方法来学习地理知识, 有一部分学生是 用其他方法来记忆地理知识, 少部分学生依靠死记硬背的方法来记忆地理知识。

3. 8. 做读图填空练习时是否会选择合适的地图查阅

从图 1（h）中可以看出, 楚雄师院附中、龙江中学和楚雄州民族中学高中生在做读图填空练 习时会选择合适的地图查阅的比例远远大于不会选择合适的地图查阅。从这里可以看出大部 
分学生有良好的做题用图习惯, 但也说明大部分学生在读图填空练习时对地图的依赖性也很 高, 因此, 教师在教学过程中应适当的培养学生做题用图的良好习惯, 但要杜绝学生过度依 赖地图。

2.9. 是否可以不借助教材在地图册上完成填图

从图 1 (i) 中可以看出, 楚雄市城区高中生在地图册填图时能不借助教材的学生很少, 这说 明学生对教材的知识不熟悉, 因此在做地图册填图时需要依赖教材。

\section{4. 改善楚雄市城区高中生地理读图能力现状的对策与建议}

4.1. 激发学习动机, 培养学生对地图的兴趣

在学习过程中, 学习的兴趣极大地影响着学生对学习内容包括地图的学习程度。在教学过程 中, 教师可以通过举一些有关生活的一些实例, 让学生感觉到地图与我们的生活息息相关、

无处不在, 从而引起学生在生活中对地理现象的观察进一步引起学生的学习兴趣, 引导学生 自觉关注身边的地理图像, 激发学生对地理读图学习的兴趣。此外, 教师也应该引导学生去 观察、关注地理现象, 阅读一些有关的地理杂志、地理电视节目等等, 提供知名地理科普网 站, 丰富学生视野, 激发学习和探索地理科学兴趣, 建立学生正确的地理科学观。

4.2. 对学生的地图基础知识加以巩固

楚雄市城区的高中地理课程首先根据教学要求, 对学生的欠缺 “补救” 内容进行选择, 适当 的 “补救” 内容对学生的地理读图的学习及能力有极大地帮助。其次, 楚雄市城区高中教师 可以根据教学的进度, 由浅入深地做到对地理图像知识过度, 这是学生容易接受的教学方式。 最后, 在教学过程中, 楚雄市城区高中教师可以利用课堂时间将基础知识穿插在其中, 不仅 让学生学习新的知识, 也加强并巩固旧的、基础性的知识, 组织学生的课堂互动, 地图知识 较好的学生帮助地图知识基础较差的学生, 这样巩固了学生的基础知识还能培养学生对地图 知识学习的积极性, 改善了学生地理读图的能力。

4.3. 培养学生良好的用图习惯

楚雄市城区高中教师在教学过程中应注重引导学生地图在日常生活中的重要性, 提醒学生时 刻关注生活中的小细节, 比如: 火车站、汽车站都会有当地小地图帮助游客或其他不熟地方 路线的人们找到到达目的地方法和路线等。教师还可以在学校及社会允许的条件下组织学生 到野外进行实践活动, 到大自然中探索地理知识, 尽可能多了解发生的地理现象, 让同学了 解到课堂中所学的地理知识对现实生活是有用的, 现实生活中又充满了很多的地理现象等着 去发现, 激发学生的好奇心和积极性, 开阔学生的地理视野, 培养学生的用图习惯, 提高学 生的地图能力。

4. 4. 培养学生地图的应用能力

在调查过程中, 发现楚雄市城区高中生的地图应用能力薄弱, 因此在地理教学中, 应该培养 学生的地理读图能力, 楚雄市城区高中课堂教学过程中首先要让学生认识地图, 在认识地图 的基础上教学生会读图、用图, 紧接着教学生绘图, 最后, 教学生会分析图, 这是学生读图 能力培养的一个必经过程, 有顺序地、循序渐进地培养学生的地图应用能力是非常重要的。 在教学过程中, 应该对学生进行有步奏地培养学生的地理读图能力, 根据识图、读图、用图、 绘图和析图着手培养学生地图的应用能力。

4. 5. 给学生创建良好的地理地图学习环境

无论是在哪里学习, 一个好的学习环境对学生的学习帮助是不容小䖋的, 学生接受教育的主 要场所是学校, 因此, 楚雄市城区高中学校应该给予学生良好的学习环境, 不断完善地理试 验室及实验器材, 多多鼓励学生动手自制地图模型, 并进行展示或者评比, 提高学生的自信 心。除了学校, 家庭和社会也应该给学生创造有利的学习环境, 有利于学生在好的环境下学 习。 


\section{5. 致谢}

本文得到云南省卓越青年教师特殊培养项目 (自然地理学) 资助。

\section{参考文献}

[1] 李玲. 高中地理教学中学生读图能力训练研究 [D]. 东北师范大学, 2007.

[2] 李寿欣．认知方式理论在教育上的应用 $[\mathrm{J}]$. 应用心理学，1995. 18 (6).

[3] 周鹏. 用多元智能理论做指导, 优化高中地理课堂教学 [J]. 学苑教育, 2015, 06: 16-17.

[4] 宋金敏. 论高中地理教学中学生地图能力存在的问题与培养方法 [D]. 河北师范大学, 2012.

[5] 陈澄. 地理学习论 [M]. 上海:华东师范大学出版社, 2001：30.

[6] 刘利科. 创新教育与中学地理课堂教学模式的构建 [D]. 长沙：湖南师范大学, 2004.

[7] 杨素君. 大理州白族地区高中生地理学习现状及教学对策的研究 [D]. 云南师范大学, 2005.

[8] 韩玉清. 地理学习评价的观察法研究 [J]. 中学地理教学参考, 2004，05：49.

[9] 马红英. 高中地理读图能力的培养 [J]. 文理导航 (下旬)，2012，07：63-64.

[10] 林翠建. 地理教学中如何培养学生的读图分析能力 [J]. 中学教学参考, 2014, 07: 114.

\section{Acknowledgement}

This research was financially supported by Yunnan Excellent Young Teacher Project (natural geography).

\section{References}

[1] ling Li. Study on Map Reading Skill Training of High School Students, Northeast Normal University, 2007.

[2] Shouxin Li. Application of Cognitive Style Theory in Education. Applied Psychology. 1995. No. 6, pp. 366-367+379.

[3] Peng Zhou. Optimizing High School Geography Teaching with Multiple Intelligences Theory. Academy of Education. 2015. No. 6, pp. 16-17.

[4] Jinmin Song. The Problems and Training Methods of the High School Students Map Reading Ability. Hebei Normal University. 2012.

[5] Chen Chen. Geography Teaching Theory, Shanghai Education Press, 2007.

[6] Like Liu. Creative Education and Middle School Geography Teaching Model, Hunan Normal University. 2004.

[7] Sujun Yang. Study on Current Situation of Geography Learning of High School Students in Dali Prefecture. Yunnan Normal University. 2005.

[8] Yuqing Han. Study on Observation Method of Geography Learning Assessment. Teaching Reference of Middle School Geography. 2004. No. 5. pp. 49.

[9] Hongying Ma. Training of high school geography Reading Ability. Liberal Arts Navigation. 2012. No. 7. pp. 63-64. 
[10] Cuijian Lin. How to Train map analysis ability in Geography Teaching. Teaching Reference of Middle School. 2014. No. 7. pp. 114.

作者简介: 陈娇芬 (1992-), 女, 云南腾冲县人, 主要研究方向为中学地理教学, E-mai1: 1920548590@qq. com。导师:

席武俊 (1979-), 男, 云南文山市人, 副教授, 研究方向为遥感与地理信息系 统应用, E-mai1: absxwj@163.com。 